\section{Ueber den heutigen Stand der Wasserbehandlung des Typhus.}

Von

\section{Dr. Ernst Brand in Stettin.}

(Fortsetzung aus No. 4.)

Es fragt sich nun, sind diese beiden Fälle geeignet, stricte $\mathrm{zu}$ beweisen, dass bei den von Anfang ab und regelrecht nach meinen Angaben behandelten Typhusfällen es nicht zur Ulceration, sondern nur zur Infiltration der Darmdrüsen kommt?

Niemand wird bestreiten, dass beide Fälle ächte Typhen gewesen sind; ebensowenig, dass der französische den schweren zuzuzählen ist. Bei dem meinigen ist der Einwand, dass er ein leichter gewesen, bei welchem das Fehlen der Darmgeschwüre hier und da vorkommt, eher zu erwarten.

Darauf ist $\mathrm{zu}$ erwidern, dass der Verlauf nur scheinbar ein leichter war und durch die Behandlung zu einem solchen umgestaltet worden ist. Für die Schwere spricht das Hin- und Herschwanken und das irreguläre Verhalten der Temperatur, die Dauer des Fiebers, die mächtige Volumzunahme der Leber, der Milz, der Gekrösdrüsen und die mangelhafte Wirkung des Chinin. Der Einwand zerfällt also in sich selber.

Unbestreitbar ist auch das Fehlen der Darmgeschwüre in beiden Fällen. Von Mouïsset wird es direct ausgesprochen: "Nirgends gewahrt man Substanzverlust, weder alten, noch neuen " am "21. Tage, und sowohl Generalarzt Dr. Abel, wie ich, haben in unserm Falle (31. Tag) keine Ulceration, keine Narbe, keine pigmentirten Stellen, sondern nur frische Infiltration $z u$ entdecken vermocht.

Ebenso kann der etwaige Einwurf, dass in meinem Falle das Typhusproduct, 'wie man es früher sehr bezeichnend genannt hat, an einen andern Ort, als das Ileum, z. B. in den Leer- oder Grimmdarm oder auch in den Magen (wohin beim Pferde die Ablagerung normal ist) abgesetzt gewesen sein könnte, nicht aufrecht erhalten werden, denn der Grimm- und Leerdarm waren ganz gesund, und der Magen, wenn auch seltsam verändert, doch frei von acuter Erkrankung. Er war nicht grösser, wie derjenige eines Kindes von 4-6 Jahren, und der Pylorustheil durch eine circuläre Einziehung von den übrigen zwei Dritteln des Magens abgeschnürt, so dass er das Aussehen einer Sanduhr oder einer Fischblase 'hatte. Diese seltsame Misstaltung des Magens ist mir nun schon 2 Mal bei Typhustodten vorgekommen. Wodurch sie entstanden ist, was sie bedeutet, ob sie mit dem Typhus zusammenhängt, insbesondere ob sie in einem Verhältnisse steht zu den heftigen Schmerzen, über die mein Kranker in epigastrio geklagt hat, weiss ich nicht zu sagen.

Und wenn man schliesslich noch einwenden sollte, dass zwei Fälle $\mathrm{zu}$ wenig sind, um eine Frage von solcher Bedeutung endgültig zu entscheiden, so muss daran erinnert werden, dass solcher Fälle, eben weil die von Anfang ab mit Wasser behandelten Typhen nicht sterben, immer nur wenige sein und bleiben werden. Habe ich auf den meinigen doch 26 Jahre gewartet, und es ist der reine Zufall, dass auch in Lyon einer vorgekommen ist. Da sind zwei Fälle doch wohl von demselben Gewicht, wie sonst zweihundert.

Der längst und ,mit Ruhe" erwartete stricte Beweis, dass die Darmaffection bei meiner Methode der Typhusbehandlung in von Anfang $a b$ und regelrecht behandelten Fällen nur bis zur Infiltration fortschreitet und Ulceration nicht zu Stande kommt, ist somit beigebracht.

Jürgensen bestreitet in seinem neuesten Werke $S .283$ noch die Möglichkeit. „Jedenfalls ist sicher", schreibt er, ,dass Todesfälle durch Perforation oder Blutung erfolgen, und dass die anatomischen Veränderungen im Darme nachweisbar bleiben". Wenn er die erst in späterer Zeit in Behandlung genommenen Fälle im Auge hat, ist gegen die Behauptung Nichts einzuwenden. Dass bei ihnen Geschwüre und Blutungen und Perforation vorkommen können, wird Niemand bestreiten; ich auch nicht. Schliesst er dagegen auch die von Anfang ab behandelten Fälle ein oder meint er nur diese, so hat er offenbar Unrecht, und man darf hoffen, dass nach meiner obigen Mittheilung in der 2. Auflage seines Werkes der Satz anders lauten wird, um so mehr, da die Perforation bei der Wasserbehandlung überhaupt nur eine sehr seltene Erscheinung ist. In 1200 regelrecht nach meiner Methode behandelten Fällen ist nur 1 Mal der Tod durch Perforation und Peritonitis eingetreten und zwar bei Jürgensen. Der Kranke war zwar schon am 3. Tage in Behandlung gekommen, mit der regelrechten Wasserbehandlung wurde aber erst am 9. Tage begonnen, also zu spät, als dass die Complication noch hätte verhütet werden können.

Jürgensen geht offenbar zu weit, wenn er aus diesem einen Fall, der obendrein wegen des späten Beginnens der Behandlung als Gegenbeweis nicht dienen kann, - dem einzigen Fall auch, den er in 20 Jahren gesehen hat; so wie ich in 30 Jahren keinen gesehen habe, den Schluss zieht, dass meine Annahme nicht gerechtfertigt ist. Sollten bei der antipyretischen Methode Perforation und Peritonitis vorkommen, nun dann wäre das nur ein Beweis mehr, dass die Wirkungsgrösse derselben mit derjenigen der Wasserbehandlung nicht aequal ist, und dass die bei dieser gewonnenen Beobachtungen für die Wasserbehandlung nicht verwerthet werden dürfen.

Die französischen Autoren rühmen an der systematischen Wasserbehandlung primo loco die Möglichkeit, den Typhuskranken hinreichend ernähren $\mathrm{zu}$ können. Nicht allein, dass derselbe nach Vollendung des Kampfes mit dem Fieber Appetit, ja Hunger und nicht selten Heisshunger bekommt, gestattet die gleichzeitig bewirkte Beseitigung des Magen- und Darmkatarrhs auch, ihm Lebensmittel in gehöriger Abwechslung reichen zu können. Und mehr noch, - er verdaut sie auch. Wie ganz anders ist es hier, als bei der exspectativen Behandlung! Während bei der Wasserbehandlung die Zunge feucht, blass, ohne Belag, der Schlund schmerzfrei, der Magen und Darm ohne Katarrh ist, der letztere nur angeschwollene Drüsen zeigt, ist dort die Zunge braun, trocken, hart, der Speichel fehlt, das Schlingen ist schmerzhaft, der Magen und Darm zum Verdauen völlig unfähig, die Schleimhaut mit Geschwüren bedeckt, der Darm meteoristisch aufgetrieben, und bei dem Zustande soll - verlangt die Schule - der Kranke gut genährt werden. Welch ein sonderbares Verlangen!

"On peut conclure" schreiben Tripier und Bouveret, ,que, au point de vue de la perte du poids, le traitement par les bains froids présente sur tous les autres cet avantage: la perte de poids est moins forte et la période d'augmentation marche plus rapidement que la période de diminution".

Die Folge davon ist, dass der mit Wasser behandelte Typhuskranke nach der Entfieberung mit mehr Kräften in die Reconvalescenz eintritt, als der exspectativ Behandelte nach vielen Wochen besitzt. Der Erstere geht schon längst ohne Unterstützung spazieren, wo der letztere unter gleichen Verhältnissen noch, ein Bild des Jammers, im Bette liegt. Die Verdauungsorgane des mit Wasser behandelten Typhuskranken befinden sich in einem dem Normalen so nahen Zustande, dass ich mir öfter die Frage vorgelegt habe, ob es nicht am Ende gestattet sein möchte, ihm derbere Nahrung zu reichen, als die gewöhnlichen flüssigen Nahrungsmittel, die nicht hinreichen, seinen Hunger zu stillen. Mir in meiner Stellung war es nicht möglich, sie zu beantworten. Ich habe deshalb nach einer geeigneten Persönlichkeit gesucht, die im Stande wäre, sie zu lösen, aber keine gefunden. Auch der verstorbene Frerichs konnte mir eine solche nicht nennen.

Nun aber finde ich in Virchow's Archiv, 89. Band, S. 95 und 303, eine Arbeit von Hösslin: Experimentelle Beiträge zur Frage der Ernährung fiebernder Kranker, welche die Frage so weit löst, dass auch der praktische Arzt ohne Besorgniss den Typhuskranken kräftiger und ausgiebiger, wie bisher, ernähren lassen darf. Es ist in derselben nachgewiesen, dass selbst bei Fieber und bestehenden Diarrhöen, so lange dieselben keinen höheren Grad erreichen, wie es bei Typhus gewöhnlich der Fall ist, die gegebene Nahrung nahezu vollständig ausgenutzt wird, dass also kein Grund besteht, deshalb weniger Nahrung dem Fiebernden zu geben; weil sie nicht verdaut und resorbirt würde. Ist das in München bei der Liebermeister'sehen Behandlung der Fall, bei welcher von den Antipyreticis reichlich Gebrauch gemacht und der Magen verdorben wird, so wird es um so sicherer geschehen bei der Methode Brand, wo die Verdauungsorgane sich in viel besserer Verfassung befinden.

Auch die Temperatur und der Stoffwechsel scheinen durch reichlichere Nahrungszufuhr nicht wesentlich erhöht, Diarrhöen und dgl. nicht veranlasst zu werden. Hösslin hält deshalb für im Ernährungszustande herabgekommene Typhuskranke (was sie wohl Alle sind!) eine möglichst grosse Nahrungszufuhr und zwar mit concentrirten Nahrungsmitteln, wie Milch, Fleisch, Eier, Mehlspeisen etc. für direkt geboten, - Alles jedoch in möglichst verkleinertem und flüssigem Zustande. „Wer aber kräftige Nahrung zuführen will," schreibt er S. 130, "der muss nothwendig wissen, wie viel Nahrung er giebt, und muss dieselbe, wenigstens annähernd, in Zahlen ausdrüicken können. Wer nicht auf die Quantität der zugeführten Nahrung die genaueste Rücksicht nimmt, der vernachlässigt gerade das Wichtigste. Ich habe deshalb, um für den praktischen Fall die Rechnung der Gesammtzufuhr zu erleichtern, den Nährwerth einiger der bei Fieber gebräuchlichsten Nahrungsmittel zusammengestellt und zwar ausgedrückt in Calorien der Verbrennungswärme. Ein gesunder Mensch verbraucht in Ruhe täglich etwa 2400 bis 2800 Cal. Diese Grösse kann auch bei stark .herabgekommenen Menschen wohl kaum bis auf die Hälfte herabsinken; dies wäre das Aeusserste, zum Leben absolut nothwendige Maass. Bei Fieber ist der Verbrauch noch vermehrt. Ein Teller Schleimsuppe, Bouillon etc. 10-20 Cal., concentrirte Suppen 40-50 Cal., ein 
Ei 68 Cal., eine Tasse Kaffee 120 Cal., eine Semmel 140 Cal., 100 Gramm Zwieback ca. 350 Cal., ein Liter Milch 747 Cal., 100 Gramm roher Schinken 150 Cal., 100 Gramm rohes Fleisch $(=60$ Gramm Braten) 110 Cal., 100 Gramm Wein 81 Cal., 100 Gramm Cognac 360 Cal. - 6

Der Passus ist in extenso wiedergegeben, weil die Nährfrage jetzt allenthalben auf der Tagesordnung steht, und diese Angaben Höss lin's der grössesten Beachtung werth sind. Ich meine aber doch zu all diesem hinzufügen zu müssen, dass das Nothwendigste zur Ernährung ausser den Speisen - ein guter Magen ist. Wo der fehlt, nützen alle Speisen nichts. Und da man ihn mittelst der systematischen Wasserbehandlung schaffen kann, ist diese auch hierdurch geboten.

Im Uebrigen haben sowohl Glénard, Tripier und Bouveret, wie auch Vogl den Speisezettel für Typhuskranke reichlicher ausgestattet, als es noch von mir geschehen ist.

Die Ersteren geben während der Kampfperiode: Milch, Bouillon, Fleischextract, Kaffee und Thee mit Milch, $1 / 4$ Liter immer nach dem Bade, also etwa $8 \mathrm{Mal}$ täglich. Während der Zeit der relativen Apyrexie, wie sie von ihnen genannt wird, erlauben sie noch Semmel, Reiscrême, Tapioca, Chocolade; Cacao, Racahout, lait de poule, weiche Eier, Fleischsaft. Von den Eiern erlauben sie bis zu 4 Stück täglich. Von der Entfieberung ab (unter $38,5^{\circ} \mathrm{C}$ ) machen sie die Mahlzeiten am Tage reichlicher, in der Nacht seltener (wie auch ich es rathe!): Hühnerbraten, Brotschnitte in Milch oder Bouillon getaucht und, wenn das gut vertragen wird, mageren Fisch, geschmortes Kalbfleisch mit Reis, gebackenes Hirn, weisses Fleisch ohne Fett, wenig gekochtes Rindfleisch, fein gehackt oder in entfetteter Bouillon. u. s. w. Damit kann der Gesunde sich zufrieden geben, wie viel mehr der Typhuskranke.

Auch Vogl geht in seiner Diät über das von mir empfohlene Maass hinaus. Vom 1. Tage ab erhalten die typhuskranken Soldaten Kaffee, Fleischbrühe mit etwas Mehl und Ei, Milch, täglich 1 Flasche Rothwein; später noch in der Fieberperiode Bouillon mit Ei und sonst noch Eier, so dass die Kranken täglich 50 Gramm Eiweiss, 45 Gr. Fett und 200 Gr. Kohlehydrate erhalten - ,ein Nährgehalt, den man nicht badenden Kranken nicht gestatten und nicht zumuthen darf."

Nach meiner Ansicht besteht gegenwärtig die Gefahr, dass, während man früher den Typhuskranken zu knapp hielt, sie jetzt mehr gefüttert werden, als ihnen gut und nöthig ist, - dass man aus einem Extrem in das andere geräth. -

Mit den $\mathrm{Complication}$ en bei der systematischen Wasserbehandlung kann man sich kurz fassen. Bei den von Anfang ab behandelten Fällen sieht man überhaupt keine entstehen und bei den später in Behandlung genommenen viel weniger, als bei der Li ebermeister'schen oder gar der exspectativen Methode. Vogl giebt darüber interessanten Aufschluss, S. 488:

Tabelle V

Zusammenstellung der wichtigeren Complicationen in Procenten.

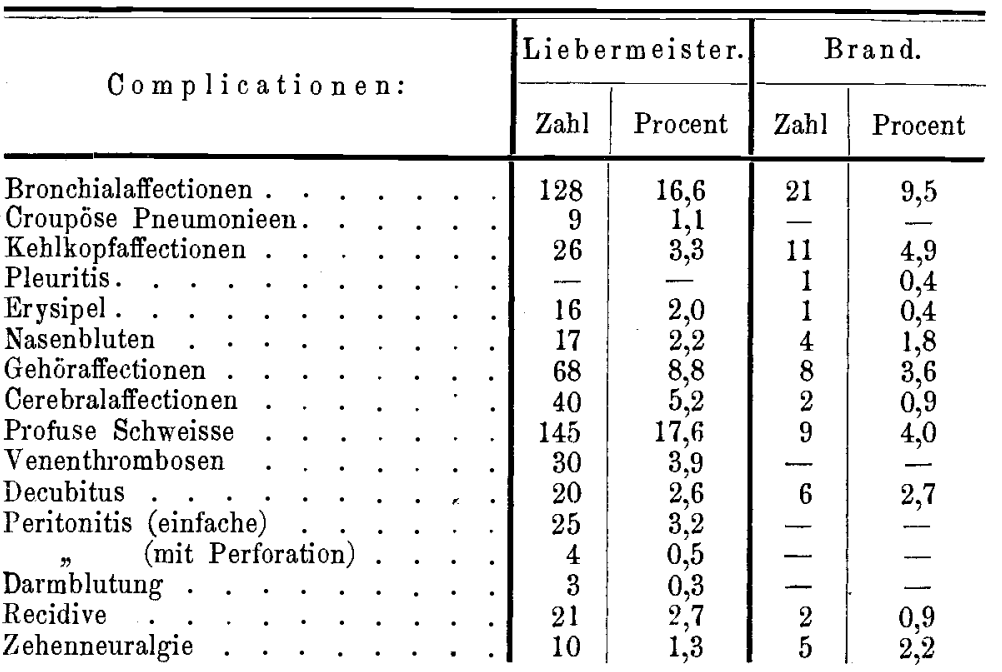

Zu den Complicationen, welche bei der Brand'schen Methode nicht beobachtet wurden, gehören auch noch Trismus, Somnolenz, Muskelzittern, Convulsionen, Harnverhaltung, Blasenkatarrh, Hämaturie, Miliaria, Endocarditis, Arythmie, Lymphgefässentzündung, Gangrän des Hodens, Gelenkentzündung.

Der Vergleich des procentualischen Vorkommens einiger wichtigeren Complicationen bei der exspectativen, Liebermeis ter's und Brand's Methode ist zu interessant, als dass er hier nicht Platz finden sollte:
Schwere Bronchialaffectionen Decubitus

Schwere Gehirnerscheinungen Darmblutung

Peritonitis mit Perforation

exspectativ
$25 \%$
$10 \%$
$25 \%$
$5,6 \%$
$9,0 \%$

Liebermeister
$16,6 \%$
$2,6 \%$
$5,2 \%$
$0,3 \%$
$0,5 \%$

Brand

$9,5 \%$

$2,7 \%$

$0,9 \%$

$0,0 \%$

$0,0 \%$

Woher die $2,7 \%$ Decubitus rühren, giebt $\mathrm{V}_{0} \mathrm{gl}$ nicht an. Alle übrigen Beobachter, die nach mir behandeln, sagen ausdrücklich, dass bei meiner Behandlung Decubitus nicht vorkommt. -

Was zum Schlusse die Indicationen und Contraindicationen bei der systematischen Wasserbehandlung anlangt, so kann man nach dem heutigen Stande der Sache sagen, dass ihre Zahl, nachdem die Furcht vor Erkältung und die Idee von der Fluxion nach dem Innern so ziemlich überwunden sind, gegen früher zwar auf ein Minimum zusammengeschrumpft, aber doch immer noch $\mathrm{zu}$ gross ist.

Vogl nimmt als Contraindication der kalten Bäder S. 499 an, 1) nur diejenigen $Z$ wischenfälle, welche dem Kranken absolute Ruhe gebieten: Peritonitis simplex und Perforation, spätere Darmblutung bei Schorfablösung und Venenthrombosen, Zustände, die ihm aber nicht vorgekommen sind; 2) die Larynx- und Pleuraaffectionen Bei ersteren badet er gar nicht, bei den letzteren wärmer, aber nur "ungern und selten, und hat das noch nie zu bereuen gehabt". Warum also?

Tripier und Bouveret stellen S. 253 als primäre Contraindicationen auf: organisches Herzleiden und chronische Phthise; als secundäre: Peritonitis und Perforation, starke Darmblutung in späterer Zeit, Pericarditis und Pleuritis im Ausgange der Krankheit. Sie fügen auch hinzu, dass, wenn man die Sterblichkeit am Typhus herabsetzen will, man, anstatt die Zahl der Contraindicationen zu vermehren, im Gegentheil suchen müsse, dieselben zu vermindern. Das sind goldene Worte, aber leider befolgen sie sie selber nicht immer. Weiter oben habe ich angegeben, dass sie bei der Pneumonie unter Umständen auf das Bad verzichten, wo dasselbe gerade dringend nöthig ist. Auch in Bezug auf die Hémorrhagie tardive et grave möchte ich Glénard beistimmen, wenn er verlangt, dass bei Darmblutung das Verfahren nicht ausgesetzt werden darf, so lange die Temperatur und die bestehenden Functionsstörungen es fordern. Wenn die Temperatur in Folge des Blutverlustes unter $39^{0} \mathrm{C}$ sinkt, wird es an und für sich Niemandem einfallen, zu baden.

(Fortsetzung folgt.) 